\title{
TRIBUTACIÓN EN COLOMBIA: LA TASA ÓPTIMA QUE MAXIMIZA EL RECAUDO TRIBUTARIO
}

\author{
Héctor David Bejarano Navarro ${ }^{1}$
}

\section{Resumen}

El presente trabajo es la experiencia en las actividades de creación, gestión, dirección y seguimiento a los estudiantes inscritos en el semillero en la línea de investigación denominada «Contabilidad y Finanzas Públicas».

Se utilizó la metodología econométrica de regresión lineal múltiple para verificar el cumplimiento de la tesis de Laffer en Colombia, la primera variable independiente corresponde a la tasa impositiva (o su logaritmo), definida como la relación entre los ingresos tributarios corrientes y el PIB corriente, para el año correspondiente; la segunda variable independiente es la tasa impositiva al cuadrado (o el cuadrado del logaritmo de la tasa impositiva); la última variable independiente es el Índice de Producción Industrial (IPI), a través del cual se pretenden recoger los efectos causados en el recaudo tributario debido al ciclo económico. La variable dependiente es el recaudo tributario por persona medido en pesos del año 2000.

Ante la problemática de crisis económica que puede terminar con una reducción del PBI en Colombia y una reducción en la recaudación del impuesto para los años venideros, los gobiernos vienen ideando diferentes mecanismos para incrementar los ingresos tributarios. Este trabajo propone dar respuesta a la siguiente pregunta ¿Qué tasa de tributo se requiere para financiar el presupuesto de gastos del gobierno central?

Como conclusión un estado moderno requiere una dosis de impuestos directos y otra de tributación indirecta haciendo un justo balance entre las dos fuentes de tributación procurando administrar mejor los tributos directos, aplicando una base suficiente para no tener que depender de la tributación indirecta que aunque es más fácil de administrar y recaudar, tiene menor capacidad distributiva del ingreso que la tributación directa.

Palabras claves: Reforma fiscal, política tributaria, impuestos, crecimiento económico..

1 Economista, especializado en estadística, magister en ciencias económicas, docente de tiempo completo 


\section{TAXATION IN COLOMBIA: OPTIMA RATE TO MAXIMIZE THE TAX REVENUES}

\section{Abstract}

The present work is the experience in building activities, management, steering and monitoring the students enrolled in the nursery in the research entitled "Accounting and Public Finance". Econometric methodology was used multiple linear regression to verify compliance with Laffer's thesis in Colombia, the first independent variable corresponds to the tax rate (or its logarithm), defined as the ratio of current tax revenues and current GDP for that year, the second independent variable is the tax rate squared (or the square of the logarithm tax rate), the last independent variable is the Industrial Production Index (IPI), through which they intend to collect the effects on tax receipts due to the economic cycle. The dependent variable is tax collections per person measured in 2000 dollars. The concern of the various governments to increase tax collections induces a party to introduce reforms to tackle deficit situations in response to increased spending by the central government, on the other hand, provide tax breaks to stimulate growth and competitiveness some sectors of the economy and result in reduced revenues and pressure on other taxpayers. The result of these tax reforms has not contributed to solving the growing deficit of the central government. This paper estimates the average tax rate that maximizes tax revenues with respect to changes in the tax rate, to generate additional resources to finance social spending aimed at poverty indices populations of concern.

Keywords: Tax reform in Colombia, tax exemption, economic growth, investment, income tax.

\section{Introducción}

Ante la problemática de crisis económica que puede terminar con una reducción del PBI en Colombia y una reducción en la recaudación del impuesto para los años venideros, los gobiernos vienen ideando diferentes mecanismos para incrementar los ingresos tributarios. Este trabajo propone dar respuesta a la siguiente pregunta: ¿Qué tasa de tributo se requiere para financiar el presupuesto de gastos del gobierno central?, mediante la aplicación de un modelo econométrico básico, aplicando el principio de parsimonia y soportado en el paper escrito por Hsing Yu (1996). ${ }^{2}$

Como objetivo general se ha propuesto el de encontrar para la economía colombiana la tasa

${ }^{2}$ El proyecto del documento fue presentado en una Conferencia de economía y finanzas de Louisiana celebrada en la Universidad de Nueva Orleans, Luisiana. Hsing examina la curva Laffer para los EE.UU. impositiva que genera para el gobierno central los máximos recaudos tributarios; dentro de los específicos se ha propuesto determinar el modelo de ajuste y definir las variables dependientes e independientes que hacen funcional el modelo econométrico.

Como conclusión al presente trabajo, un estado moderno requiere una dosis de impuestos directos y otra de tributación indirecta haciendo un justo balance entre las dos fuentes de tributación procurando administrar mejor los tributos directos, aplicando una base suficiente para no tener que depender de la tributación indirecta que aunque es más fácil de administrar y recaudar, tiene menor capacidad distributiva del ingreso que la tributación directa. 
En los trabajos hasta ahora realizados, se han utilizado diferentes enfoques para medir la parte correspondiente al PIB registrado en las cuentas nacionales y otros al aumento de la economía informal de un período a otro. Todos estos métodos tienen limitaciones (Blanchard, 2000), por falta de datos o porque utilizan hipótesis no comprobadas empírica ni teóricamente.

Un dogma neoliberal afirma que la manera más eficiente y efectiva de estimular la economía es bajando los impuestos. Como prueba de ello, varios economistas neoliberales se han referido a la experiencia de la administración del Presidente Ronald Reagan, cuando con la reducción masiva de impuestos del gobierno en 1981 fue la causa de la recuperación económica a principios de los años ochenta en EEUU.

La idea del uso de aspectos fiscales para incentivar el crecimiento económico se hizo tan fuerte que, de hecho, algunos autores afirman que es posible relacionar tasas de impuestos con variables como el crecimiento económico y el desempleo. Un estudio de este tipo fue desarrollado por Alan Auerbach y James Hines (1988). Pasados 26 años desde el trabajo revelador de Jorgenson, Peter Clark, un estudioso de la Escuela de Chicago, afirmó haber encontrado pruebas de que los beneficios tributarios a la inversión poco repercutían en las decisiones de las firmas sobre su capital (Clark y Sichel, 1993). Clark no encontró en su análisis de series de tiempo significancia en las variables que debían medir el efecto de los beneficios fiscales sobre los índices de inversión en la economía.

Debido a la controversia por los resultados de los estudios de Clark, el profesor Robert Hall tuvo oportunidad de refutar en el marco de paneles de expertos sobre el tema. Al respecto, Hall expresó su escepticismo por el uso de series de tiempo para estas mediciones, en las cuales la determinación de rezagos efectivos era compleja. Otros estudiosos del tema apoyaron a Hall. Ellos co- mentaron acerca de sus experiencias personales en la consultoría de inversiones a grandes firmas de Estados Unidos y manifestaron que los beneficios fiscales siempre eran considerados al momento de tomar una decisión de inversión estratégica y que, de hecho, en repetidas ocasiones esos beneficios eran los detonantes para la toma de una decisión de inversión.

Otro trabajo reciente que sigue un método similar al que a continuación se describe es el desarrollado por House y Shapiro (2004). En este trabajo, los autores encuentran que los incentivos tributarios pueden incrementar los niveles de capital de la economía de estado estacionario. Ello no necesariamente tiene que suceder; sin embargo, sí concluyeron que el mecanismo al menos funciona para adelantar las decisiones de inversión.

La ecuación es la siguiente: en la medida en que un país reduce sus impuestos, atrae más inversión, propicia condiciones de empleo e incrementa su actividad económica de tal modo que sus ingresos no decaen pese a que su tasa tributaria disminuye (Due, 1968). Esa correlación, conocida como la curva de Laffer, está demostrada en una serie de economías en todo el mundo.

Centralizando la posición del autor, en Colombia se tiene el sistema según el cual los grandes tributos tienen carácter nacional (renta, IVA) pero simultáneamente se han diseñado mecanismos de transferencia a entidades territoriales que han asumido responsabilidades de ejecutar determinados gastos e inversiones. La tendencia de todo el mundo con relación al impuesto de renta es la de rebajar las tarifas marginales y en 1995 se elevó al 35\%. En la actualidad hay un relativo consenso entre los teóricos fiscales en el sentido que las tarifas excesivamente altas desalientan el ahorro, el esfuerzo empresarial, fomenta la evasión, desestimulan la movilidad de capitales internacionales que se está imponiendo en el mundo. 


\section{Contenido}

La metodología a seguir es la investigación cuantitativa referida a la investigación empírica sistemática a través de técnicas estadísticas y matemáticas y el objetivo de la investigación cuantitativa es revisar si los impuestos recaudados por el gobierno nacional siguen un comportamiento parabólico (figura 1) aumentando primero, llega a un punto máximo y luego decrece. Para ello se supone que los impuestos se cobran a los ingresos del factor trabajo y/o a las ganancias que los individuos reciben de las empresas. El modelo planteado por Artur Laffer plantea ideas que pueden representarse en la curva, que relaciona niveles de recaudación en el eje vertical con la tarifa impositiva en el eje horizontal conformando una función que tiene forma de campana.

Figura 1 Curva parabólica de Laffer

CUAVA DE LAFFER

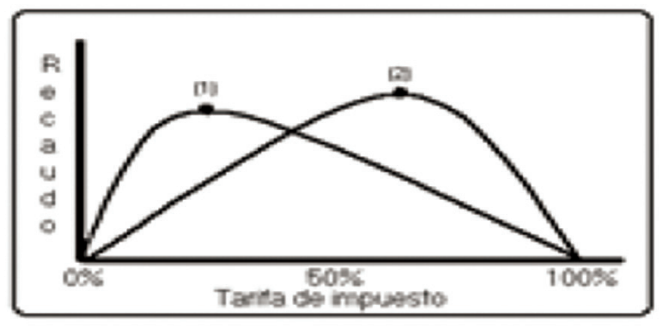

Fuente el autor

La actual estructura tributaria de Colombia ha sido consecuencia de cambios históricos en los últimos años y han traído consigo rápidos cambios suscitados por la modernización y globalización de la economía. A partir de los años 1970 América Latina comienza una reestructuración fiscal evidenciada a través del cambio en la ordenación tributaria, estos cambios comienzan a dar vía al recaudo de impuestos más por la vía indirecta que directa (Bolaños, 2010).

Aunque existen diversas escuelas de pensamiento sobre metodología econométrica, en el presente trabajo se ha utilizado la metodología tradicional clásica (Gujarati, 2000) técnica que predomina en la investigación empírica económica y en diversos campos relacionados.

Planteamiento de la teoría de hipótesis: Al relacionar los ingresos tributarios recaudos con las tasas impositivas se evidencia que los recaudos provenientes por cobro se asocian a una forma de campana, iniciativa propuesta por el profesor Arturo Laffer El objetivo es demostrar que la curva por el recaudo fiscal (Hernández, 2005) llega hasta un máximo y luego decrece, es decir, se pretende calcular el punto de inflexión o default en el que se obtiene el máximo recaudo y a partir de allí empiezan los ingresos a decrecer como producto del incremento de las tarifas impositivas (figura 2).

Figura 2. La tarifa fiscal y el ingreso tributario
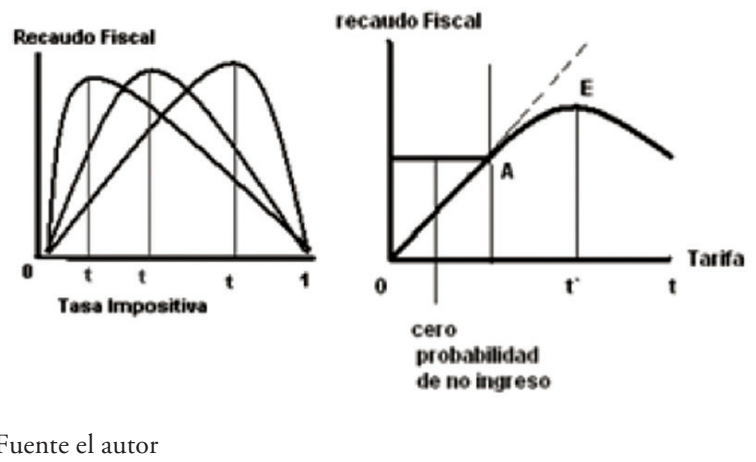

Si la tasa impositiva es la variable independiente, el recaudo tributario presenta cambios ante las variaciones en la tasa impositiva. Significa esto, que ante aumentos sucesivos en los impuestos, se incrementan los ingresos fiscales hasta un cierto nivel, a partir del cual empiezan a declinar. Este argumento depende del impacto que tengan los cambios en las tasas impositivas y del impacto que tenga el ingreso real de los agentes económicos. Se cuestiona entonces ¿Cuál es la tasa impositiva para que el recaudo tributario sea máximo? (figura 2).

La curva es una representación del valor esperado para el recaudo fiscal que percibe el gobierno. Para niveles bajos de tarifas impositivas se espera que se cumpla con los gastos 
del gobierno, y por tanto la curva tiene pendiente positiva y la probabilidad de déficit fiscal es igual a cero. No obstante, una vez las tarifas sobrepasan el nivel «A» (panel derecho de la figura 2) la probabilidad de déficit aumenta y por tanto los ingresos esperados empiezan a aumentar menos que proporcional que los gastos. En el punto E (panel derecho de la Imagen 2) los ingresos esperados alcanzan el máximo y a partir de ese punto empiezan a descender, la curva entonces, es cóncava tal como se mostró en la figura 1.

El problema de recaudar vía impuestos de renta es que tasas muy altas generan desestímulos a la creación de negocios o a la formación de capital. Para entender mejor la situación se reflexiona: ¿cuánto sería el recaudo estatal con una tasa de renta del $0 \%$ ? La respuesta es obvia, el recaudo sería nulo. Entonces pregúntese: ¿cuánto sería el recaudo con una tasa de renta del $100 \%$ ? La respuesta también es lógica, el recaudo también sería cero. Como resulta lógico, nadie iniciaría una actividad empresarial sabiendo que no podrá disfrutar de sus utilidades. Este racionamiento simple permite evidenciar que la tributación por renta exhibe una curva de Laffer. Este fenómeno de sustitución (trade off) entre tasas tributarias y recaudos tributarios es llamado usualmente curva de Laffer, por el economista que popularizó su existencia, Arthur Laffer, al final de los años setenta, (González, 2005)

Especificación matemática del modelo: A pesar de existir inicialmente una relación positiva entre la tasa impositiva y el recaudo por este concepto, los tratadistas no especifican de manera precisa una relación funcional entre las dos variables (Guisan, 1997). Para especificar el modelo se ha seguido la teoría de la curva de la tasa impositiva sobre la renta expuesta por Hsing (1996). La comunidad científica económica no ha tenido reparos en su formulación por tal razón se ha seguido su postulado. El docu- mento presentado por Hsing examina la curva de Laffer para los EE.UU. sobre la base de datos de series de tiempo durante el período de 1959-1991.

Para optimizar el modelo en la economía colombiana y después de muchas aproximaciones infructuosas se ha logrado plantear una función cuadrática (Buchanan, 1982) tomando como variables las tasas impositivas y el índice de producción industrial (IPI) que recoge la actividad productiva de la rama industrial (extractiva, manufacturera, producción, distribución de energía eléctrica, agua, gas) que refleja la evolución de la cantidad y la calidad del producto, eliminando la influencia de los precios en el período. La función cuadrática para el modelo se expresa mediante la siguiente función:

$$
I T R_{t}=f\left(T_{t}, T_{t}^{2}, I P I_{t}\right)
$$

Donde la variable dependiente es:

$I T R_{t}=$ Recaudo tributario real per cápita medido en pesos del año 2000 en el período t. Es una variable que soporta los imperfectos de la carga tributaria que soportan los colombianos, dadas las diferencias de ingreso existentes entre los estratos de la población

Variables independientes: Recogen la presión tributaria como resultado de modificar las tarifas en las diferentes reformas realizadas en el período:

$T_{t}$ = Tasa impositiva en el período t.

IPIt = Índice de producción Industrial en el período $t$.

Especificación del modelo econométrico: Para especificar el modelo econométrico (Greene, 1998) se utiliza la metodología de regresión li- 
neal múltiple para el período 1980-2010. Las formas funcionales empleadas y estimadas corresponden a la lineal y semi logarítmica, sin tener razones para preferir cualquiera de ellas (Hsing, 1996). La primera variable independiente corresponde a la tasa impositiva (o su logaritmo), definida como la relación entre los ingresos tributarios nominales y el PIB nominal para el año correspondiente; la segunda variable independiente es la tasa impositiva al cuadrado (o el cuadrado del logaritmo de la tasa impositiva); la última variable independiente es el Índice de Producción Industrial (IPI), a través del cual se pretenden recoger los efectos causados en el recaudo tributario debido al ciclo económico.

Siguiendo el modelo formulado por Hsing en su artículo publicado en 1996 en The Journal of Socio-Economics y teniendo en cuenta el concepto de la teoría de la curva y su optimización, la forma funcional específica es lineal en los parámetros y no en las variables y se define de la siguiente forma:

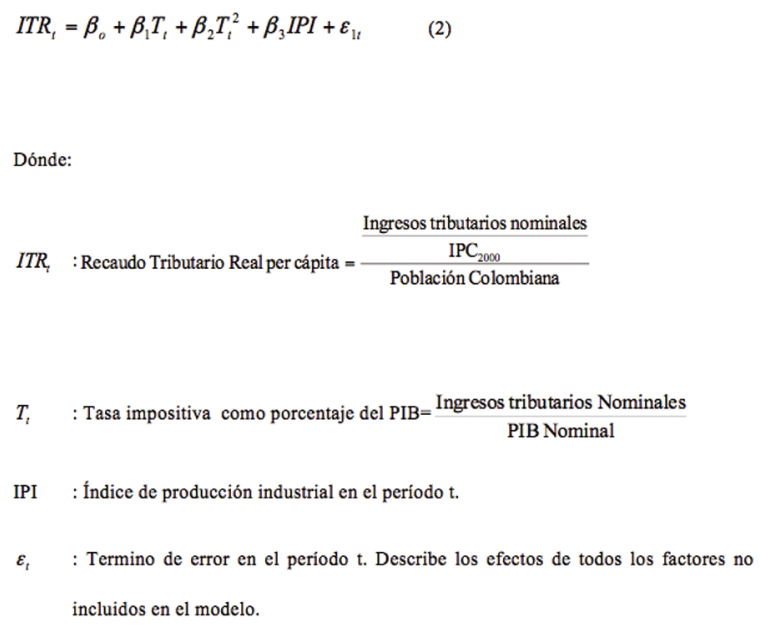

$\beta_{0}, \beta_{1}, \beta_{2}, \beta_{3}$ : Parámetros de regresión a estimar por el método de Minimos Cuadrados Ordinarios (MCO).

La ecuación (2) se utiliza para evidenciar la curva de segundo grado y probar si los parámetros de regresión $\beta_{1}$ y $\beta_{2}$ son significativos y $\beta_{2}$ es negativo. Si los coeficientes $\beta_{1}$ y $\beta_{2}$ son significativos y positivos, la función cuadrática tiene una forma de $U$, situación contraria al concepto de la curva en forma de campana.

Para estimar el impacto de las reformas tributarias sobre el recaudo se utilizó un modelo de serie de tiempo con datos que corresponden a operaciones efectivas reportadas por la DIAN desde 1970. Se prefirió iniciar la serie a partir de 1980 porque el índice de Producción Industrial (IPI) se encuentra disponible en el Dane a partir de esa fecha. El período escogido obedece a la disponibilidad de datos, período dentro del cual se encuentran las reformas tributarias que introdujeron cambios significativos en las tarifas y bases gravables, o que buscaban disminuir la evasión o aumentar la eficiencia en el recaudo. El Índice de Producción Industrial (IPI) mide la evolución mensual de la actividad productiva de las ramas industriales, es decir, de las industrias extractivas, manufactureras y de producción y distribución de energía eléctrica, agua y gas. Este indicador refleja la evolución conjunta de la cantidad y de la calidad, eliminando la influencia de los precios.

\section{Resultados y estimación de los parámetros del modelo econométrico}

En todas las regresiones ajustadas se llevó a cabo el proceso de verificación de supuestos mediante las pruebas Breusch-Pagan (hetero cedasticidad), Breusch-Godfrey (auto correlación), y Kolmogorov-Smirnov (normalidad), Ilegando a la conclusión que los residuales estimados en todas las regresiones son normales, homocedásticos y auto correlacionados, por lo cual se aplica el método de corrección de mínimos cuadrados generalizados. Los resultados empíricos son el resultado del uso y manejo del programa de Stata y empleo de las herramientas econométricas para el logro de los resultados contenidos en la siguiente tabla: 
Tabla 1. Resultados de la estimación (valores p en paréntesis)

\begin{tabular}{|c|c|c|c|c|c|c|c|}
\hline $\begin{array}{l}\text { Forma } \\
\text { funcional } \\
\mathrm{N}^{\circ}\end{array}$ & Intercepto & Tasa & Tasa $^{2}$ & IPI & $\begin{array}{l}\mathrm{R}^{2} \\
\text { ajustado }\end{array}$ & $\square$ & $\begin{array}{l}\text { Tasa } \\
\text { óptima }\end{array}$ \\
\hline Log-lin & $\begin{array}{l}3,902 \\
(0,000)\end{array}$ & $\begin{array}{l}17,660 \\
(0,000)\end{array}$ & $\begin{array}{l}-34,462 \\
(0,000)\end{array}$ & $\begin{array}{l}0,004 \\
(0,000)\end{array}$ & 0,994 & 0,683 & $25,62 \%$ \\
\hline
\end{tabular}

Fuente: El autor

La única forma funcional que cumple con las condiciones restrictivas para la ecuación (2) arriba descrita es la log - lin con un tasa optima de impuesto de $25.62 \%$ como respuesta a la función planteada. Antes de efectuar las regresiones se realizaron las pruebas de cointegración o de raíz unitaria a efecto de no encontrar una relación espuria entre las variables. Se realizó la prueba de contraste Dickey-Fuller (1979) de raíces unitarias rechazando la hipótesis de no existencia de cointegración en las series utilizadas. En todas las regresiones de las formas funcionales se llevó a cabo el proceso de verificación y prueba de las hipótesis, para llegar a concluir que los residuales estimados son normales, homocedásticos y autocorrelacionados, haciendo uso de los test correspondientes.

\section{Discusión.}

Una vez diseñado, conceptualizado y ejecutado el modelo y realizado el ajuste según la batería de índices de ajuste y la verificación que no hay diferencia estadísticamente significativa entre el modelo conceptualizado y el modelo teórico, se procede a pronosticar con el modelo de mejor ajuste en su forma funcional log-lin que se expresa mediante la siguiente función:

\section{Re caudoFiscal $(t)=3,902+17,66\left(\right.$ Tasa $\operatorname{Im}$ positiva $\left.\left._{t}\right)-34,462(\text { Tasa } \operatorname{Im} \text { positiva })_{t}\right)^{2}+0,004($ IPI $)$}

El ajuste al modelo cuadrático es muy bueno con un valor $R^{2}$ igual a 0,994 considerado alto y con una significatividad conjunta e individual de los parámetros muy alta. Igualmente se ha evidenciado la estabilidad estructural de los parámetros al ser eficientes

El estudio ha demostrado que para la economía colombiana es evidente la existencia de una relación entre las variables macroeconómicas recaudo tributario real per cápita y la tasa impositiva como porcentaje del PIB, mediante la utilización de una muestra de 31 observaciones para el período 1980-2010.
Al maximizar la ecuación cuadrática mediante el empleo de la relación $\left(-\beta_{2} / \beta_{2}\right)$, se determina la tasa impositiva como porcentaje del PIB del $25.62 \%$ nivel donde el recaudo fiscal real per cápita logra su máxima expresión al hacerse óptimo.

Si las variaciones al recaudo tributario como porcentaje del PIB es superior a este nivel, el recaudo tributario real per cápita empieza a declinar dependiendo del impacto que tenga el cambio en la tasa impositiva y del impacto en el ingreso real de los diferentes agentes económicos. 
Figura 3 -Optimización del ingreso tributario del Gobierno Central

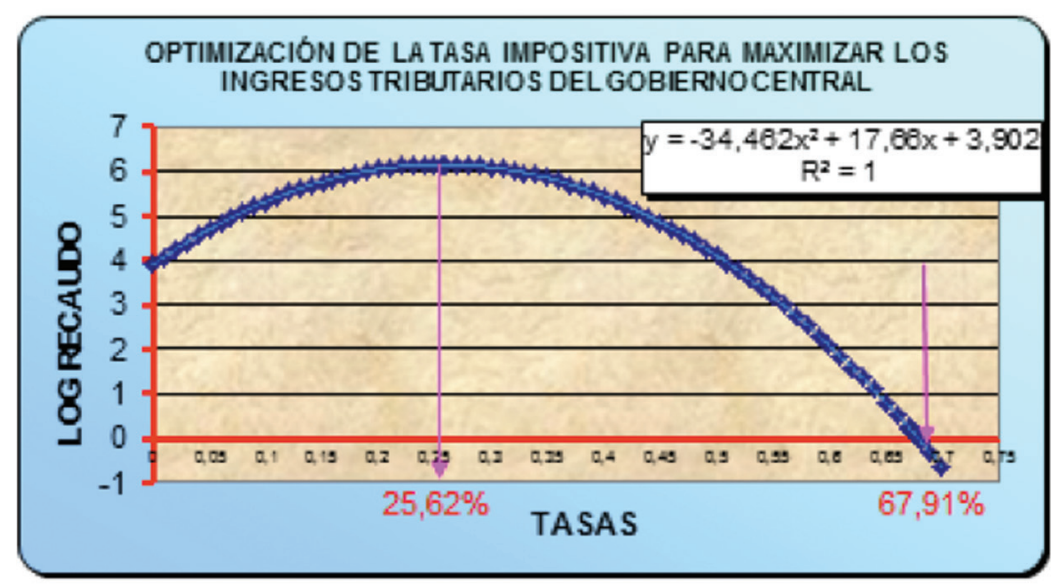

Fuente el autor

Una tasa impositiva superior al $68 \%$ el gobierno central no percibe ingreso tributario alguno puesto que el impacto marginal al recaudo tributario real per cápita es negativo.

Según los resultados obtenidos, el recaudo tributario aumenta a un ritmo creciente hasta alcanzar un máximo donde la tasa impositiva es equivalente al $25.6 \%$, a partir de este guarismo la contribución del recaudo tributario es decreciente.

\section{Recomendaciones}

Colombia es una de las naciones del mundo con mayor desigualdad (Garay, 2002) que se expresa en que el $20 \%$ de los hogares más ricos concentran el $52 \%$ de los ingresos y el quintil con mayores ingresos devenga 26.3 veces más que el quintil más pobre. Además el $1.1 \%$ de los propietarios de la tierra en el país tienen más del $55 \%$ del territorio cultivable y explotable. $\mathrm{Pa}-$ rece que en este contexto es razonable utilizar la política fiscal para desarrollar una verdadera política social alrededor de la inclusión social, de la lucha contra la pobreza y del crecimiento con el concurso de las políticas públicas.

Mi criterio es que en la actualidad hay un relativo consenso entre los teóricos fiscales en el sentido que las tarifas excesivamente altas desalientan el ahorro, el esfuerzo empresarial, fomenta la evasión, desestimulan la movilidad de capitales internacionales que se está imponiendo en el mundo.

\section{Conclusiones}

Las siguientes son las conclusiones logradas al obtener la optimización de la función y del análisis a los ingresos recaudados por el gobierno central:

En particular, para el caso colombiano se puede observar que aplicar una tasa tributaria sostenida del $26 \%$ en promedio debe conducir a alcanzar un óptimo de recaudo para el gobierno central. La razón natural por la cual se espera que esto suceda ha sido estudiada desde el punto de vista de cómo actúa una menor tarifa impositiva.

Si la tasa impositiva se aumentan a partir del $10 \%$, la función es inelástica y significa que ante incrementos sucesivos a partir de dicho guarismo, los ingresos no crecen en la misma proporción.

Con márgenes de tasas impositivas superiores al $26 \%$ la situación es preocupante puesto que el ingreso recaudado por la contribución disminuye significativamente hasta no percibir ingreso alguno por concepto de impuesto si este en promedio es igual o mayor a $67.9 \%$. 
En Colombia se tiene el sistema según el cual los grandes tributos tienen carácter nacional (renta, IVA) pero simultáneamente se han diseñado mecanismos de transferencia a entidades territoriales que han asumido responsabilidades de ejecutar determinados gastos e inversiones. La tendencia de todo el mundo con relación al impuesto de renta es la de rebajar las tarifas marginales.

\section{Referencias}

Auerbach, A. y Hines, J. (1988). Investment tax incentives and frequent tax eforms. The American Economic Review , 78 (2), 211-216.

Buchanan, James M., y Dwight R. Lee, (1982). Politics, Time, and the Laffer Curve, The Journal of Political Economy, Vol.90, No 4: 816 - 819.

Blanchard, Oliver. (2000). Macroeconomía. Madrid: Pearson Education.

Bolañoz Muñoz Jhoanna. (2010). Una aproximación a la evolución de los impuestos en Colombia. Fundación Dialnet.

Clark, P. y Sichel, D. (1993). Tax incentives and equipment investment. Brooking Papers on Economic Activity, (1), 317-348

Clavijo, Sergio, (2005). Tributación, equidad y eficiencia en Colombia: Guía para salir de un sistema tributario amalgamado, http://www.banrep.gov.co [consultado marzo 2007]

Due Jhon, (1968). Análisis Económico de los impuestos, $2^{\mathrm{a}}$ edi, REIG Jorge Enrique, traductor, Editorial El Ateneo.

González, A. 2005. Recaudo del gobierno en presencia de deducciones tributarias basándose en las inversiones realizadas por las empresas: una sencilla aplicación al caso colombiano. Bogotá: Universidad Javeriana.

Guisán María del Carmen. (1997). Econometría. Madrid: Mc Graw Hill.

Gujarati Damodar. (2000). Econometría. Diseño de modelos econométricos. Cap 13. México: Mc Graw Hill. Quinta edición.

Greene William H. (1998). Análisis Econométrico.. México: Prentice Hall.
Garay, Luis Jorge. (2002). Colombia Entre la Exclusión y el Desarrollo: Propuestas para la Transición al Estado Social de Derecho. Bogotá: Contraloría General de la Republica.

Hernández Rodríguez Isidro. (2005). Teoría y política fiscal. Capítulo tercero. Teoría y política tributaria. Primera edición. Bogotá: Universidad Externado de Colombia.

House, C. y Shapiro, M. (2004). Temporary investment tax incentives: theory with evidence from bonus depreciation. Recuperado el 25 de noviembre del 2005, de http:// www.personal.umich.edu

Hsing Hu. (1996). Estimating the Laffer curve and Policy Implications. Southeastern Lousiana University. Journal of Socio - Economics, volumen 25, № 3: 395 - 401.

Iregui B., Ana María; Melo B., Ligia \& Ramos F., Jorge. (2005).El impuesto predial en Colombia: Factores explicativos del recaudo. Revista de Economía, Universidad del Rosario.

Junguito, Roberto y Rincón, Hernán. (2004). La Política Fiscal en el Siglo XX en Colombia. Borradores de Economía, No 318, Banco de La República.

kenneth J Arrow, A: K: Sen y Cotarro Zuzumura, (1977). Una dificultad en el concepto de bienestar social. New York: Thompson.

Krugman Paul. (2012). Economía Internacional. México: Prentice Hall. 9 Edición.

Lora, Eduardo y Mauricio Cárdenas. (2006). Las reformas de las instituciones fiscales en América Latina. Documento de Trabajo \# 559. Washington, D. C.: Banco Interamericano de Desarrollo.

Lucas, R. (1990). "Supply-side Economics: An Analytical Review". Oxford Economic Papers, 42(2), 293-317.

Modiglianni Franco. (1977). La controversia monetarista. Trimestre Económico. № 206 abril/Junio de 1985.

Musgrave Richard. (1981). Teoría de Hacienda Pública, Madrid, Edit. Instituto de Estudios Fiscales.

Ocampo, José Antonio. (2004). Reconstruir El Futuro. Globalización, Desarrollo y Democracia en América Latina. Bogota: Norma-Naciones Unidas.

República de Colombia. Presupuesto General de la Nación 1974 - 2010.

Rosas Aniceto, Roberto Santillán. (1962). Teoría General de las Finanzas Públicas y el Caso de México. México D.F.: Escuela Nacional de Economía. 
Stiglitz, Joseph. (1986). La Economía del Sector Público. Barcelona: Antoni Bosch Editor.

Stiglitz, Joseph. (2004). "The Parties" Flip-Flops on Deficit Spending: Economics or Politics. The economists' voice, article 2. 2004

Sen, Amartya. (1999). Conferencia pronunciada en el "Círculo de Economía» de Barcelona. Publicado en La Factoría, No.8.

Taylor, Lance. (1986). Modelos Macroeconómicos para Los Países en Desarrollo. México D.F: Fondo de Cultura Económica.

Taylor, Lance. (1998.) La explicación didáctica del resultado de SEN se encuentra en Elección social y desigualdad económica. Cuadernos de Economía, № 29, juliodiciembre, Bogotá, Universidad Nacional de Colombia,

Zarate Ambroz, Michael. (2011). Impuestos Directos e Indirectos. México. 